\title{
Monitoring Packet Loss Impact in IPTV and 3DTV Receivers
}

\author{
Jesús Gutiérrez, Pablo Pérez, Fernando Jaureguizar , Julián Cabrera, and Narciso García
}

\begin{abstract}
Considering a scalable video quality monitoring architecture to detect transmission errors at households, we propose a technique to detect packet losses in IPTV and Side-bySide 3DTV and evaluate their impact on the perceived quality.
\end{abstract}

\section{INTRODUCTION}

Monitoring the quality of multimedia services in broadcasting systems offers interesting advantages to the end users. From a better design of the network to an adaptive billing of the services depending on the perceived quality, the applications of quality measurement are oriented to provide the best Quality of Experience (QoE) to the consumers.

Among the several methods to evaluate the quality of audiovisual contents, only a limited amount could be used for real-time monitoring applications. Specifically, objective NoReference (NR) metrics are needed [1], since there is no availability of the original content in these scenarios, and subjective assessment tests are not practical.

The quality of the audiovisual content could suffer degradations in every step of the broadcasting chain (e.g. capture, coding, transmission, etc.). However, minimum quality requirements should be satisfied when a real delivery system is deployed, especially concerning the quality of the original material and the encoding process. Therefore, the present work is focused on detecting and evaluating the impact on video quality of typical transmission errors in packet-based networks. This type of errors is mainly caused by packet losses, jitter, and service outage, causing effects such as macroblocking, choppy transitions, and video freeze [2].

Furthermore, the broadcasting of new audiovisual content to the consumers should be followed by the adaptation of the QoE monitoring techniques. For example, nowadays the first channels of 3DTV are being offered to the end users. These approaches are based on the existing infrastructure (i.e. coders, networks, decoders, etc.) used to deliver conventional video, such as Side-by-Side (SbS) 3D video. Therefore, although several new factors influence the QoE in 3DTV services, some aspects could be analyzed by evolving 2D metrics.

In this paper we propose a monitoring technique to detect impairments in the video quality caused by packet losses in IPTV and SbS 3DTV systems. In Section II, the architecture is described in detail. In Section III, experimental results and their correlation with subjective evaluations are presented. Finally, conclusions of the work are presented in Section IV.

\section{PROPOSED ARCHITECTURE}

The aim of the proposed quality monitoring architecture is to act as a probe connected to the Set-Top Box (STB) at the end user side to detect various transmission errors that impair the QoE perceived by the consumers. The system is composed by modules like the one shown in Fig. 1, and each module is used to analyze one specific event. Firstly, a detection process is carried out providing a qualitative description of the impairment, which in some cases is more representative than subjective scores. For example, in the case of video freeze it would indicate the duration of the distortion. Secondly, the detected event is mapped to subjective quality values, such as Mean Opinion Scores (MOS), taking into account their impact on the perceived quality. The input of each module could be either the bitstream of encoded video or the decoded frames. When various modules are used, the global output of the probe is the maximum distortion among the different outputs. Finally, it is worth noting that a monitoring window should be set to obtain periodic measures of the quality of the video. In fact, a period of 6 seconds of video is considered.

The work of this paper focuses on the development of one module to detect video packet loses and evaluate their impact. Usually, when video packets are lost, the error concealment algorithm of the decoder replaces the lost macroblocks with the corresponding ones of the previous frame. This usually causes blocking effects degrading the video quality. The proposed method gets the decoded video from the output of the STB to analyze these effects in IPTV and SbS 3DTV.

The detection of these impairments could be done by the analysis of the intensity differences of the pixels around the macroblock boundaries. In fact, an algorithm based on the metric proposed in [3] is used. The mean value of the differences between the rows and columns of adjacent macroblocks is compared, with the differences between the rows and columns within each macroblock. This detection process provides a qualitative output consisting on the number of frames and the percentage of the frame distorted.

After detecting the lost macroblocks, the impact of the macroblocking effect is estimated by using a Just Noticeable Distortion (JND) model. The proposed metric is based on existing pixel-based JND models [4], however, the evaluation of the distortions is carried out considering macroblocks

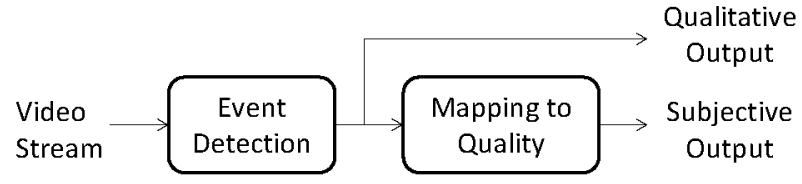

Figure 1. Module of the proposed architecture design. 
instead of individual pixels. Luminance, texture and temporal thresholds are computed, based on background luminance, the luminance variability in the neighborhood, and luminance of the previous frame, respectively. They are combined as shown in (1) to determine the perceptibility of the macroblocking, which is obtained in terms of JNDs as in (2), where $L_{M B}$ is the mean luminance of the analyzed macroblock and $L_{N}$ is the same concept considering the eight neighbor macroblocks:

$$
\begin{gathered}
\mathrm{T}=\max \left(\mathrm{T}_{\text {lum }} \cdot \mathrm{T}_{\text {temp }}, \mathrm{T}_{\text {tex }} \cdot \mathrm{T}_{\text {temp }}\right) \\
\mathrm{JND}_{2 \mathrm{D}}=\left[\operatorname{mean}\left(\mathrm{L}_{\mathrm{MB}}\right)-\operatorname{mean}\left(\mathrm{L}_{\mathrm{N}}\right)\right] / \mathrm{T}
\end{gathered}
$$

In the case of $\mathrm{SbS} 3 \mathrm{D}$ video, this model is extended considering the computation of the JND of the corresponding macroblocks in both stereo views, as in (3). This allows the evaluation of the binocular rivalry felt by the observers when transmission errors affect differently each stereo view, causing that very different content is shown in corresponding regions (see Fig. 2). The total distortion for a macroblock in SbS video is given by the expression in (4).

$$
\begin{gathered}
\mathrm{JND}_{\mathrm{SbS}}=\left[\operatorname{mean}\left(\mathrm{L}_{\mathrm{MB}-\mathrm{LEFT}}\right)-\operatorname{mean}\left(\mathrm{L}_{\mathrm{MB}-\mathrm{RIGHT}}\right)\right] / \mathrm{T} \\
\mathrm{JND}_{\mathrm{T}}=\mathrm{JND}_{2 \mathrm{D}} \cdot \mathrm{JND}_{\mathrm{SbS}}
\end{gathered}
$$

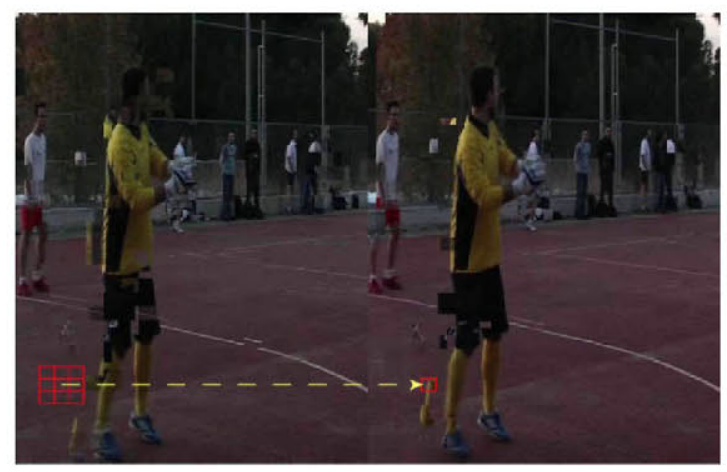

Figure 2 . SbS frame affected by macroblocking

The mean of the JND values of the macroblocks of an impaired frame is computed to obtain a global JND for that frame. In addition, the JND values of the frames are pooled to obtain the JND for the video segment during the monitoring window. A Minkowski sum is applied as stated in (5), where $N$ is the number of frames.

$$
\mathrm{D}=\left(\sum_{\mathrm{i}}^{\mathrm{N}} \mathrm{JND}_{\mathrm{T}, \mathrm{i}}^{2}\right)^{1 / 2}
$$

These values provided by the metric should be mapped to subjective scores, such as MOS. Therefore, a non-symmetric logistic function is used. Its expression is shown in (6), where $a$ and $b$ are the parameters of the function, $u_{\min }$ and $u_{\max }$ are the minimum and maximum subjective values obtained.

$$
\operatorname{MOS}_{\mathrm{p}}=\mathrm{u}_{\min }+\frac{\mathrm{u}_{\max }-\mathrm{u}_{\min }}{1+(\mathrm{D} / \mathrm{a})^{1 / \mathrm{b}}}
$$

\section{RESULTS}

To test the performance of the proposed monitoring technique, segments of 6 seconds of 3 different video sources were used (for the 3D case, the stereoscopic versions of two of these sequences were used). These segments were distorted with different patterns and durations of macroblocking. The quality estimations of the proposed metric were compared with the results obtained in a subjective assessment test. A detailed explanation of the generation of the test sequences and the subjective methodology could be found in [5].

In Table 1 the performance results of the metric are shown in terms of Pearson linear correlation coefficient, normalized Root Mean Square Error, and outlier ratio. In addition, the number of segments used in the fitting process and the number of tests segments are shown. The results show an acceptable prediction of the quality perceived by the end users when video is distorted by packet losses, both for IPTV and 3DTV.

TABLE 1

PERFORMANCE OF THE METRIC

\begin{tabular}{cccccc}
\hline \hline $\begin{array}{c}\text { Test } \\
\text { Video }\end{array}$ & $\begin{array}{c}\text { Training } \\
\text { Segments }\end{array}$ & $\begin{array}{c}\text { Test } \\
\text { Segments }\end{array}$ & $\begin{array}{c}\text { Pearson } \\
\text { Correlation }\end{array}$ & RMSE & $\begin{array}{c}\text { Outlier } \\
\text { Ratio }\end{array}$ \\
\hline 2D & 19 & 62 & 0.7715 & 0.1482 & 0.1852 \\
3D & 12 & 47 & 0.8305 & 0.1695 & 0.1712 \\
\hline \hline
\end{tabular}

\section{CONCLUSION}

Monitoring the video quality at households allow service providers to know the QoE perceived by the viewers in their homes, and act in order to satisfy their expectations. In this paper, we consider a scalable monitoring architecture that permits the detection of typical transmission errors, and provides subjective estimations and qualitative outputs useful for service providers. A module of this architecture is presented, which detects video packet losses from the decoded frames in IPTV and SbS 3DTV receivers. The impact of these impairments in the perceived video quality is evaluated by means of a pixel-based JND model working with macroblocks instead of individual pixels. The results provided by the metric show an acceptable estimation of the perceived video quality.

Future work will be focused on the improvement of the JND model, and the development of the architecture with new modules to detect more transmission errors and complement the one proposed, for instance with bitstream-based metrics like the one presented in [6], that will improve the overall performance.

\section{REFERENCES}

[1] S. S. Hemami and A. R. Reibman, "No-reference image and video quality estimation: Applications and human-motivated design," Signal Processing: Image Communication, vol. 25, no. 7, pp. 469-481, Aug. 2010.

[2] G. W. Cermak, "Consumer opinions about frequency of artifacts in digital video," IEEE Journal of Selected Topics in Signal Processing, vol. 3, no. 2, pp. 336-343, Apr. 2009.

[3] A. R. Reibman and D. Poole, "Characterizing packet-loss impairments in compressed video," in IEEE International Conference on Image Processing, vol. 5, pp. 77-80, 2007.

[4] W. Lin and C.-C. Jay Kuo, "Perceptual visual quality metrics: A survey," Journal of Visual Communication and Image Representation, vol. 22, no. 4, pp. 297-312, May. 2011.

[5] J. Gutiérrez, P. Pérez, F. Jaureguizar, J. Cabrera, and N. García, "Subjective evaluation of transmission errors in IPTV and 3DTV," Int. Conf. on Visual Communications and Image Processing, 2011 (in press).

[6] P. Pérez, J. Macías, J. J. Ruiz, and N. García, "Effect of packet loss in video Quality of Experience," Bell Labs Technical Journal, vol. 16, no. 1, Jun. 2011. 